\title{
Part II - Dorsal wrist pain and swelling post trauma 2
}

\author{
John Vedelago • Elizabeth Dick • Miny Walker • \\ Afshin Alavi • Monica Khanna • Wladyslaw Gedroyc
}

Published online: 22 January 2013

(C) ISS 2013

\section{Diagnosis}

Traumatic lipohaematoma of the extensor tendon sheaths (compartments 2-4) post distal radial fracture.

\section{Discussion}

In the same way that identification of a lipohaemarthrosis alerts the reading radiologist to the presence of an intraarticular fracture, the visualisation of a lipohaematoma of the tendon sheath after trauma may herald the presence of fracture extending into an osseous groove for a tendon. It also indicates injury to the tendon sheath, and raises the possibility of associated tendon injury, which may warrant further assessment of the affected tendon with magnetic resonance imaging.

A 29-year-old man presented post cyclist vs car accident. CT showed a comminuted distal radial fracture. As shown in Fig. 3 of the case presentation, a fracture line cleaved completely through the base of Lister's tubercle (larger arrow) with disruption of the floor of the fourth compartment by an acute angled sharp fracture fragment (smaller arrow). Fracture lines extended into the grooves for extensor pollicis longus (3rd extensor compartment) and extensor digitorum (4th extensor compartment). A separate fracture passed into the grooves for extensor carpi radialis longus and brevis (2nd extensor compartment). The edges of the

The case presentation can be found at doi: 10.1007/s00256-012-1569-0

J. Vedelago $(\bowtie) \cdot$ E. Dick $\cdot$ M. Walker $\cdot$ A. Alavi $\cdot$ M. Khanna $\cdot$

W. Gedroyc

Department of Radiology, St. Mary's Hospital, Praed Street,

W2 1NY, London, UK

e-mail: john.vedelago@imperial.nhs.uk fracture fragments were sharply angulated and disrupted the osseous grooves for the 3rd and 4th compartment tendons. The medullary cavity of the distal radius appeared open to the tendon sheaths of both the 3rd and 4th extensor compartments. An oblique sagittal fracture line was also seen extending from the volar surface of the radius through the articular surface. A small lipohaemarthrosis of the radiocarpal joint was present. Further distally, within the tendon sheaths of the second, third and fourth extensor compartments prominent fat-fluid levels within the tendon sheaths were seen, accounting for the gross soft tissue swelling seen on the radiograph. The lipohaematoma was most voluminous within the distal third and fourth compartment sheaths, and comparatively small in the second compartment sheaths. The average Hounsfield Unit density of the upper (and, by volume, larger) layer within the tendon sheaths was approximately -90 , consistent with the presence of fat.

Magnetic resonance imaging obtained 4 days after the injury showed fat-fluid layers within the non-dependent aspect of the sheaths of the second, third and fourth compartments. A layer of high T1 signal within the nondependent aspect of the sheaths (part 1 figure 2 - arrow), which completely saturated on $\mathrm{T} 2$ fat suppression and corresponded to the position of the $-90 \mathrm{HU}$ density material seen on the $\mathrm{CT}$, was present, confirming the presence of fat. The distal radial fracture is also evident on this image. There was focal irregular high T2 signal seen along the deep radial side surface of tendons of the extensor digitorum and extensor pollicis longus adjacent to the sharp fracture edges, consistent with tendon abrasion, but no frank split or rupture was seen. The dependent portion of the lipohaematoma showed signal characteristics consistent with a mixture of fluid and blood. Figure 4 of this article shows a fracture line evident in the distal radius, which appears to have disrupted the tendon sheath and is opening into the floor of the fourth 


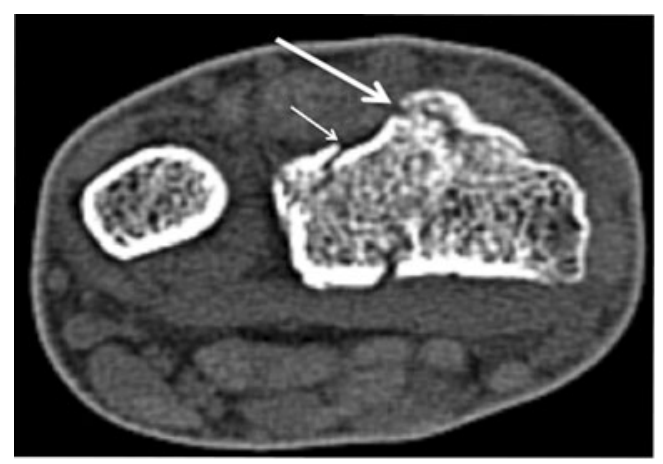

Fig. 3 Fracture lines were seen to cleave through Lister's tubercle (larger arrow) and into the floor of the fourth compartment (smaller arrow)

compartment. The floor appears disrupted and there is focal abnormal signal involving the adjacent tendons. The patient's injury was managed non-operatively with immobilization and the swelling resolved 4 weeks post-injury.

Lipohaematoma of the tendon sheath is theorised to occur when a sharp fracture fragment tears through the thin synovial layers of a tendon sheath or a subjacent fracture through a groove causes a closed disruption of a sheath during highvelocity trauma, thereby exposing bone marrow directly to the potential space within the sheath and allowing for direct extrusion of marrow to occur. Imaging features have been previously described [1], although the entity has not been previously named, or documented involving the 4th extensor compartment. It is thought most likely to occur after fracture through an osseous groove because of the close proximity of the sheath here to cortical bone, the relatively delicate constitution of the sheath and its comparative anatomical restriction within a fibro-osseous groove.

Histologically, the synovial cell-lined tendon sheath is a thin structure composed of two distinct cell layers and a scant surrounding fibrous tissue support network [2]. The structures deep to the tendon sheath of the fourth extensor

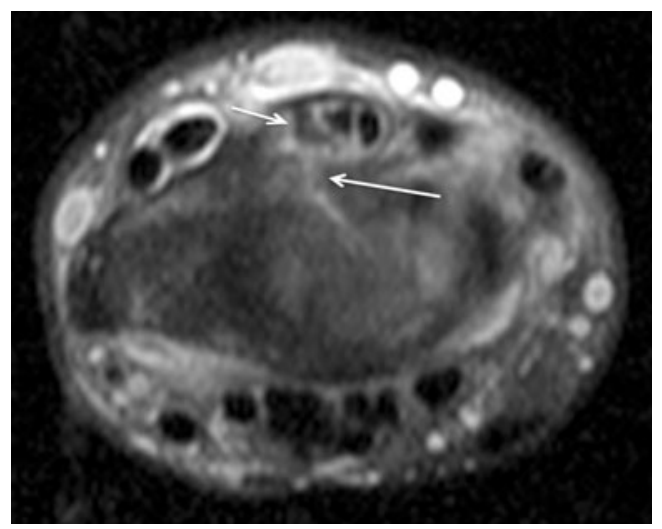

Fig. 4 Axial T2 fat-saturated image through the distal radius shows a fracture line evident in the distal radius (long white arrow), which appears to have disrupted the tendon sheath and is opening into the floor of the fourth compartment. The floor appears disrupted and there is focal abnormal signal involving the adjacent tendons (short arrow) compartment are thin, and comprise a basal mesotendon [3], the periosteum only in its proximal half [4], the posterior interosseous nerve [5], as well as numerous small nutrient arteries.

The finding of a lipohaematoma is of potential clinical significance for several reasons. Multiple orthopaedic authors have suggested that the presence of haematoma within the tendon sheath [6-8] post fracture predisposes to rupture owing to ischaemic complications. The observation of fat within a tendon sheath post fracture should alert the radiologist to the presence of fracture that has likely pierced or disrupted the tendon sheath in a groove. The tendon itself may have been injured, and if clinically indicated, MRI may be useful to further evaluate the structural integrity of the tendon.

A possible, but less likely alternative explanation for the presence of a fat-fluid level within the tendon sheath is that the lipid tracked from a lipohaemarthrosis within an adjacent joint via an interconnecting synovial communication or foramen. This is, however, considered a less likely explanation as the MRI appeared to show disruption of the floor of the compartment sheath with a fracture line extending into the sheath. Further, the majority of fat in case 1 was seen in the extensor digitorum longus tendon sheaths. Normal communication between the third or fourth compartment and the radiocarpal joint is not described. A normal foramen exists, however, in the vast majority of people between the sheaths of the EPL and the ECRL and ECRB at the wrist [9-11], which may explain the small amount of fat seen within the second compartment.

Primary lipomatous lesions of the tendon sheath are extremely rare and several imaging features differentiate lipohaematoma from other causes of fat within the tendon sheath. In the setting of trauma, a fat-fluid level within the sheath, with an adjacent fracture extending into an osseous groove for a tendon is characteristic of the condition. Lipoma of a tendon sheath at the wrist has been infrequently described [12] and appears as a well-circumscribed entity that should not demonstrate a fat-fluid level. Similarly, lipoma arborescens of the tendon sheath at the hand and wrist $[13,14]$ or foot $[15]$ is a very rarely reported pathology that does not exhibit distinct fat-fluid levels.

Conflicts of interest The authors declare that they have no conflict of interest.

\section{References}

1. Le Corroller T, Parratte S, Zink J, Argenson J, Champsaur P. Floating fat in the wrist joint and in the tendon sheaths. Skeletal Radiol. 2010;39:931-3. 
2. O'Connell J. Pathology of the synovium. J Clin Pathol. 2000;114:773-84.

3. Schmidt H, Lanz U. Distal forearm, wrist and dorsum of the hand. In Surgical anatomy of the hand. Stuttgart: Thieme. p. 228.

4. Cooney W. The wrist: diagnosis and operative treatment. Philadelphia: Lippincott Williams and Wilkins. p. 36.

5. Ilyas A. Surgical approaches to the distal radius. Hand. 2011;6(1):8-17.

6. Engkvist O, Lundborg G. Rupture of the extensor pollicis longus tendon after fracture of the lower end of the radius - a clinical and microangiographic study. Hand. 1979;11(1):76-86.

7. Gelb R. Tendon transfer for rupture of the extensor pollicis longus. Hand Clin. 1995;11(3):411-22.

8. Bonatz E, Kramer T, Masear V. Rupture of the extensor pollicis longus tendon. Am J Orthop. 1996;25(2):118-22.

9. Cvitanic O, Henzie G, Adham M. Communicating foramen between the tendon sheaths of the extensor carpi radialis brevis and extensor pollicis longus muscles: imaging of cadavers and patients. Am J Radiol. 2007;189(5):1190-7.
10. Zbrodowski A, Gajisin S, Grodecki J. Intersynovial communication between the tendon of the extensor pollicis longus and extensor carpi radialis brevis muscles. J Hand Surg. 1985;10(2):162-4.

11. Schmidt H, Lahl J. Studies on the tendinous compartments of the extensor muscles on the back of the human hand and their tendon sheaths. Gegenbaurs Morph Jahr. 1988;134(2):155-73.

12. Vekris M, Lykissas M, Beris A. Median nerve compression secondary to lipoma arising from flexor tenosynovium: a case report. Hand Surg. 2007;12(2):83-6.

13. Siva C, Brasington R, Totty W, Sotelo A, Atkinson J. Synovial lipomatosis (lipoma arborescens) affecting multiple joints in a patient with congenital short bowel syndrome. J Rheumatol. 2002;29(5):1088-92.

14. Hill G, Phyo N. Extra-articular lipoma arborescens of the hand: an unusual case report. J Hand Surg Eur. 2011;36(5):422-3.

15. Babar S, Sandison A, Mitchell A. Lipoma arborescens at the ankle. Skeletal Radiol. 2008;37:75-7. 